Supporting Information

\title{
Aircraft Measurement of Organic Aerosols over China
}

GEHUI WANG ${ }^{1, \dagger}$, KIMITAKA KAWAMURA ${ }^{1, *}$, SHIRO HATAKEYAMA ${ }^{2}$, AKINORI TAKAMI $^{2}$, HONG LI ${ }^{3}$ AND WEI WANG ${ }^{3}$

${ }^{1}$ Institute of Low Temperature Science, Hokkaido University, Kita 19, Nishi 8, Kita-ku, Sapporo 060-0819, Japan

${ }^{2}$ National Institute for Environment Studies, 16-2 Onogawa, Tsukuba, Ibaraki 305-8506, Japan

${ }^{3}$ Chinese Research Academy of Environmental Sciences, Beijing 100012, China

${ }^{\dagger}$ Present address: School of the Environment, Nanjing University, Nanjing 210093, China; Email address: gehuiwang@yahoo.com.cn

*Corresponding author: kawamura@lowtem.hokudai.ac.jp, Ph: 81-11-706-5457; Fax: 81-11706-7142

Revised version

Summary of the supporting information

(1) Table S1. Concentrations of single organic compounds in PM2.5 aerosols collected by aircraft over China.

(2) Figure S1. Flight tracks during the three campaigns.

(3) Page number of the supporting section including this cover sheet is 4 . 


\begin{tabular}{|c|c|c|c|c|c|c|c|c|c|c|c|c|}
\hline \multirow[b]{2}{*}{ Compound } & \multicolumn{4}{|c|}{$\begin{array}{c}\text { east coast } \\
\text { winter } \\
\end{array}$} & \multicolumn{4}{|c|}{$\begin{array}{l}\text { inland } \\
\text { summer }\end{array}$} & \multicolumn{4}{|c|}{$\begin{array}{l}\text { inland } \\
\text { spring }\end{array}$} \\
\hline & $\min$ & $\max$ & mean & std & $\min$ & $\max$ & mean & std & $\min$ & $\max$ & mean & std \\
\hline \multicolumn{13}{|l|}{ I. $n$-Alkanes } \\
\hline $\mathrm{C}_{16}$ & nd & 3.2 & 0.3 & 1.0 & nd & nd & nd & nd & nd & 2.8 & 0.2 & 0.7 \\
\hline $\mathrm{C}_{17}$ & 2.9 & 10.9 & 5.2 & 2.1 & nd & nd & nd & nd & nd & 61.8 & 16.6 & 20.6 \\
\hline $\mathrm{C}_{18}$ & 6.1 & 30.5 & 15.5 & 6.2 & nd & 8.8 & 3.2 & 3.3 & nd & 66.1 & 16.8 & 21.6 \\
\hline $\mathrm{C}_{19}$ & 8.9 & 68.2 & 34.7 & 13.9 & 3.7 & 29.8 & 15.5 & 7.5 & nd & 25.2 & 6.7 & 7.6 \\
\hline $\mathrm{C}_{20}$ & 8.9 & 61.7 & 35.1 & 14.6 & 10.4 & 59.5 & 31.9 & 13.6 & 4.0 & 29.7 & 12.5 & 6.8 \\
\hline $\mathrm{C}_{21}$ & 7.2 & 29.8 & 17.0 & 6.9 & 17.7 & 88.2 & 52.4 & 20.2 & 11.8 & 69.6 & 29.6 & 16.9 \\
\hline $\mathrm{C}_{22}$ & 2.4 & 17.3 & 9.2 & 4.6 & 9.2 & 63.5 & 30.2 & 14.6 & 10.0 & 158 & 48.9 & 37.7 \\
\hline $\mathrm{C}_{23}$ & 1.9 & 18.8 & 8.4 & 5.7 & 4.0 & 36.3 & 16.8 & 9.1 & 6.0 & 116 & 30.7 & 28.1 \\
\hline $\mathrm{C}_{24}$ & 1.6 & 13.2 & 6.1 & 3.9 & 2.5 & 21.4 & 11.5 & 6.2 & 2.5 & 37.6 & 10.0 & 9.0 \\
\hline $\mathrm{C}_{25}$ & 2.2 & 16.2 & 7.4 & 4.3 & 2.0 & 26.9 & 10.6 & 7.5 & nd & 16.6 & 4.7 & 4.3 \\
\hline $\mathrm{C}_{26}$ & nd & 11.8 & 4.0 & 3.4 & 1.4 & 21.5 & 8.4 & 6.2 & nd & 6.0 & 2.3 & 1.9 \\
\hline $\mathrm{C}_{27}$ & nd & 9.2 & 3.4 & 2.9 & 1.1 & 21.7 & 7.8 & 6.2 & nd & 8.7 & 3.3 & 2.6 \\
\hline $\mathrm{C}_{28}$ & nd & 7.8 & 2.5 & 2.5 & 0.8 & 18.5 & 5.2 & 4.9 & nd & 5.3 & 1.5 & 1.6 \\
\hline $\mathrm{C}_{29}$ & nd & 9.3 & 3.1 & 3.1 & 1.2 & 15.7 & 4.7 & 3.9 & nd & 45.4 & 11.4 & 13.1 \\
\hline $\mathrm{C}_{30}$ & nd & 6.1 & 1.4 & 1.9 & 0.6 & 10.2 & 2.7 & 2.5 & nd & 8.1 & 1.5 & 2.4 \\
\hline $\mathrm{C}_{31}$ & nd & 7.2 & 1.9 & 2.3 & 0.7 & 9.0 & 2.5 & 2.1 & nd & 8.2 & 1.8 & 2.6 \\
\hline $\mathrm{C}_{32}$ & nd & 4.1 & 0.8 & 1.2 & nd & 5.0 & 1.0 & 1.3 & nd & 1.5 & 0.2 & 0.4 \\
\hline $\mathrm{C}_{33}$ & nd & 3.0 & 0.5 & 1.1 & nd & 4.4 & 0.5 & 1.2 & nd & 2.1 & 0.2 & 0.6 \\
\hline $\mathrm{C}_{34}$ & nd & 1.6 & 0.1 & 0.4 & nd & 2.8 & 0.3 & 0.7 & nd & nd & nd & nd \\
\hline Subtotal & 44 & 316 & 157 & 69 & 62 & 349 & 205 & 86 & 57 & 615 & 199 & 149 \\
\hline \multicolumn{13}{|l|}{ II. Fatty acids } \\
\hline $\mathrm{C}_{9: 0}$ & 3.3 & 18.7 & 8.3 & 4.4 & nd & nd & nd & nd & nd & 23.7 & 3.2 & 7.0 \\
\hline $\mathrm{C}_{10: 0}$ & 2.8 & 8.8 & 5.5 & 1.7 & 7.6 & 31.5 & 17.8 & 8.7 & nd & 18.3 & 6.8 & 5.6 \\
\hline $\mathrm{C}_{11: 0}$ & nd & 1.7 & 0.4 & 0.7 & 0.0 & 13.4 & 5.3 & 3.6 & nd & nd & nd & nd \\
\hline $\mathrm{C}_{12: 0}$ & 6.7 & 20.4 & 12.6 & 3.9 & 6.9 & 64.3 & 27.0 & 14.9 & nd & 21.5 & 9.1 & 7.5 \\
\hline $\mathrm{C}_{13: 0}$ & nd & 3.2 & 1.9 & 0.7 & nd & 8.3 & 2.2 & 2.2 & nd & nd & nd & nd \\
\hline $\mathrm{C}_{19: 0}$ & nd & 1.6 & 0.5 & 0.6 & nd & 2.0 & 0.6 & 0.7 & nd & nd & nd & nd \\
\hline $\mathrm{C}_{20: 0}$ & nd & 5.8 & 2.6 & 1.8 & nd & 6.1 & 2.6 & 1.6 & nd & 1.6 & 0.2 & 0.5 \\
\hline $\mathrm{C}_{21: 0}$ & nd & 2.8 & 0.6 & 0.8 & nd & 0.2 & 0.1 & 0.1 & nd & nd & nd & nd \\
\hline $\mathrm{C}_{22: 0}$ & nd & 11.5 & 2.8 & 3.0 & nd & 3.4 & 1.0 & 1.1 & nd & 3.9 & 0.6 & 1.2 \\
\hline $\mathrm{C}_{23: 0}$ & nd & 11.7 & 1.8 & 3.0 & nd & 1.8 & 0.1 & 0.5 & nd & 4.6 & 0.3 & 1.2 \\
\hline $\mathrm{C}_{24: 0}$ & nd & 19.9 & 4.7 & 5.8 & nd & 5.7 & 2.2 & 1.8 & nd & 7.0 & 1.0 & 2.1 \\
\hline $\mathrm{C}_{25: 0}$ & nd & 3.7 & 1.2 & 1.3 & nd & nd & nd & nd & nd & 3.3 & 0.2 & 0.9 \\
\hline $\mathrm{C}_{26: 0}$ & nd & 9.9 & 2.4 & 2.9 & nd & 3.0 & 0.3 & 0.8 & nd & 9.6 & 0.7 & 2.6 \\
\hline $\mathrm{C}_{27: 0}$ & nd & 2.2 & 0.3 & 0.6 & nd & 1.0 & 0.1 & 0.3 & nd & 1.5 & 0.1 & 0.4 \\
\hline$C_{28: 0}$ & nd & 10.7 & 1.4 & 3.0 & nd & 6.4 & 0.6 & 1.8 & nd & 18.6 & 1.3 & 5.0 \\
\hline $\mathrm{C}_{29: 0}$ & nd & 4.0 & 0.3 & 1.0 & nd & nd & nd & nd & nd & 2.6 & 0.2 & 0.7 \\
\hline $\mathrm{C}_{30: 0}$ & nd & 17.4 & 1.6 & 4.4 & nd & nd & nd & nd & nd & 14.6 & 1.0 & 3.9 \\
\hline $\mathrm{C}_{31: 0}$ & nd & nd & nd & nd & nd & nd & nd & nd & nd & nd & nd & nd \\
\hline $\mathrm{C}_{32: 0}$ & nd & 1.3 & 0.1 & 0.3 & nd & nd & nd & nd & nd & nd & nd & nd \\
\hline $\mathrm{C}_{34: 0}$ & nd & 8.7 & 0.7 & 2.1 & nd & nd & nd & nd & nd & nd & nd & nd \\
\hline$C_{16: 1}$ & 4.5 & 19.6 & 9.2 & 4.2 & nd & 63.1 & 17.6 & 18.2 & nd & 7.2 & 0.5 & 1.9 \\
\hline $\mathrm{C}_{18: 1}$ & 3.1 & 12.4 & 6.8 & 2.8 & nd & 49.8 & 19.9 & 15.2 & nd & 14.1 & 1.5 & 4.1 \\
\hline $\mathrm{C}_{22: 1}$ & nd & 15.5 & 2.6 & 5.2 & nd & nd & nd & nd & nd & nd & nd & nd \\
\hline Subtotal & 26 & 160 & 68 & 35 & 30 & 223 & 97 & 55 & nd & 97 & 27 & 29 \\
\hline \multicolumn{13}{|l|}{ III. Sugars } \\
\hline Levoalucosan & 1.6 & 161 & 37.8 & 46.8 & 2.2 & 116 & 24.4 & 34.1 & 4.2 & 213 & 43.8 & 59.3 \\
\hline Arabitol & nd & 1.7 & 0.3 & 0.5 & nd & 0.3 & 0.1 & 0.1 & nd & 1.1 & 0.2 & 0.4 \\
\hline Fructose & 3.8 & 71.1 & 16.8 & 16.6 & nd & 2.4 & 0.6 & 0.7 & nd & 5.4 & 0.6 & 1.6 \\
\hline Glucose & 10.3 & 142 & 34.7 & 31.1 & 1.5 & 8.9 & 4.4 & 2.1 & 0.8 & 20.1 & 5.7 & 5.0 \\
\hline Mannitol & nd & 9.5 & 1.9 & 2.6 & nd & 0.5 & 0.1 & 0.2 & nd & 3.1 & 0.4 & 0.9 \\
\hline Inositol & nd & 7.7 & 1.6 & 1.9 & nd & 1.5 & 0.3 & 0.5 & nd & 1.0 & 0.2 & 0.3 \\
\hline Sucrose & 1.0 & 74.2 & 15.6 & 17.6 & 0.5 & 2.8 & 1.3 & 0.7 & nd & 2.1 & 0.4 & 0.7 \\
\hline Trehalose & nd & 2.7 & 0.6 & 0.7 & nd & 1.4 & 0.4 & 0.4 & nd & 1.4 & 0.3 & 0.4 \\
\hline Subtotal & 39 & 384 & 109 & 83 & 6 & 123 & 31 & 25 & 8 & 222 & 52 & 63 \\
\hline
\end{tabular}




\begin{tabular}{|c|c|c|c|c|c|c|c|c|c|c|c|c|}
\hline \multirow{3}{*}{$\begin{array}{l}\text { Table S1. Continued } \\
\text { Compound }\end{array}$} & \multicolumn{4}{|c|}{ east coastal } & \multicolumn{4}{|c|}{ Inland } & \multicolumn{4}{|c|}{ inland } \\
\hline & \multicolumn{4}{|c|}{ winter } & \multicolumn{4}{|c|}{ summer } & \multicolumn{4}{|c|}{ spring } \\
\hline & $\min$ & $\max$ & mean & std & $\min$ & $\max$ & mean & std & $\min$ & $\max$ & mean & std \\
\hline IV. Polyacids & & & & & & & & & & & & \\
\hline Glyceric acid & 2.8 & 22.8 & 7.3 & 4.9 & 1.4 & 20.1 & 6.6 & 5.2 & 1.0 & 46.7 & 10.2 & 11.3 \\
\hline Malic acid & 8.0 & 113 & 47.3 & 28.2 & 2.9 & 42.6 & 18.4 & 12.4 & 2.4 & 82.4 & 19.2 & 21.1 \\
\hline Tartaric aicd & nd & nd & nd & nd & nd & 6.1 & 2.7 & 2.1 & nd & 10.1 & 2.3 & 3.4 \\
\hline Citric acid & 1.0 & 37.7 & 6.5 & 8.6 & nd & 0.8 & 0.1 & 0.2 & nd & nd & nd & nd \\
\hline Subtotal & 21 & 120 & 61 & 27 & 5 & 65 & 28 & 18 & 4 & 136 & 32 & 25 \\
\hline \multicolumn{13}{|l|}{ V. Lignin \& Resin } \\
\hline Vanillic acid & nd & 2.8 & 0.5 & 0.7 & 0.1 & 2.1 & 0.6 & 0.6 & nd & nd & nd & nd \\
\hline Syringic acid & nd & 1.1 & 0.2 & 0.3 & nd & 0.1 & 0.0 & 0.0 & nd & nd & nd & nd \\
\hline Dehydroabietic acid & 4.5 & 13.1 & 8.4 & 2.6 & 5.7 & 41.2 & 19.1 & 9.9 & 0.4 & 1.7 & 0.8 & 0.4 \\
\hline Subtotal & 4 & 13 & 9 & 3 & 6 & 42 & 20 & 10 & 0.4 & 1.7 & 0.8 & 0.4 \\
\hline \multicolumn{13}{|l|}{ VI. Sterols } \\
\hline Cholesterol & 3.8 & 64.9 & 27.4 & 15.9 & 2.8 & 28.0 & 9.1 & 5.9 & 0.0 & 13.2 & 6.1 & 3.5 \\
\hline$\beta$-Sitosterol & nd & 2.7 & 0.5 & 0.9 & nd & nd & nd & nd & nd & nd & nd & nd \\
\hline Subtotal & 4 & 65 & 28 & 16 & 3 & 28 & 9 & 6 & 0 & 13 & 6 & 4 \\
\hline \multicolumn{13}{|l|}{ VII. PAHs } \\
\hline Naphthalene & 0.0 & 0.2 & 0.1 & 0.1 & nd & nd & nd & nd & nd & nd & nd & nd \\
\hline 2-Methylnaphthalene & 0.0 & 33.5 & 5.7 & 9.4 & nd & nd & nd & nd & nd & nd & nd & nd \\
\hline 1-Methylnaphthalene & 0.0 & 0.2 & 0.1 & 0.1 & nd & nd & nd & nd & nd & nd & nd & nd \\
\hline Biphenyl & 0.0 & 0.5 & 0.1 & 0.1 & nd & nd & nd & nd & nd & nd & nd & nd \\
\hline 2,6-Dimethylnaphthalene & 0.0 & 5.4 & 0.8 & 1.8 & nd & nd & nd & nd & nd & nd & nd & nd \\
\hline Acenaphthene & 0.0 & 0.3 & 0.0 & 0.1 & nd & nd & nd & nd & nd & nd & nd & nd \\
\hline 2,3,5-Trimethylnaphthylene & 0.0 & 4.8 & 0.3 & 1.1 & nd & nd & nd & nd & nd & nd & nd & nd \\
\hline Fluorene & 0.0 & 0.4 & 0.1 & 0.2 & nd & nd & nd & nd & nd & nd & nd & nd \\
\hline 2-Methylfuorene & 0.0 & 0.8 & 0.3 & 0.3 & nd & nd & nd & nd & nd & nd & nd & nd \\
\hline Dibenzothiophene & 0.1 & 0.9 & 0.4 & 0.2 & nd & nd & nd & nd & nd & nd & nd & nd \\
\hline Phenanthrene & 1.1 & 5.1 & 2.5 & 1.3 & nd & nd & nd & nd & nd & nd & nd & nd \\
\hline Anthracene & 0.0 & 0.9 & 0.2 & 0.3 & nd & nd & nd & nd & nd & nd & nd & nd \\
\hline Fluoranthene & 0.7 & 6.8 & 2.5 & 1.9 & nd & nd & nd & nd & nd & nd & nd & nd \\
\hline Pyrene & 0.3 & 4.0 & 1.4 & 1. & nd & nd & nd & nd & nd & nd & nd & nd \\
\hline Benzo $(b)$ fluorene & 0.0 & 1.4 & 0.3 & 0.5 & nd & nd & nd & nd & nd & nd & nd & nd \\
\hline 1,1'-Binaphthalene & 0.0 & 0.0 & 0.0 & 0.0 & nd & nd & nd & nd & nd & nd & nd & nd \\
\hline Benz(a)anthracene & 0.0 & 1.7 & 0.5 & 0.5 & nd & nd & nd & nd & nd & nd & nd & nd \\
\hline Chrysene / Triphenylene & 0.0 & 4.0 & 1.1 & 1.1 & nd & nd & nd & nd & nd & nd & nd & nd \\
\hline Benzo(b)fluoranthene & 0.0 & 14.1 & 3.2 & 4.5 & nd & nd & nd & nd & nd & nd & nd & nd \\
\hline Benzo $(k)$ fluoranthene & 0.0 & 2.9 & 0.5 & 0.9 & nd & nd & nd & nd & nd & nd & nd & nd \\
\hline Benzo(e)pyrene & 0.0 & 2.6 & 0.6 & 0.8 & nd & nd & nd & nd & nd & nd & nd & nd \\
\hline Benzo(a)pyrene & 0.0 & 1.7 & 0.5 & 0.6 & nd & nd & nd & nd & nd & nd & nd & nd \\
\hline Perylene & 0.0 & 0.4 & 0.1 & 0.1 & nd & nd & nd & nd & nd & nd & nd & nd \\
\hline 9,10-Diphenylanthracene & 0.0 & 0.1 & 0.0 & 0.0 & nd & nd & nd & nd & nd & nd & nd & nd \\
\hline Indeno[123-cd]pyrene & 0.0 & 3.9 & 1.0 & 1.1 & nd & nd & nd & nd & nd & nd & nd & nd \\
\hline Dibenz $(a, h)$ anthracene & 0.0 & 0.5 & 0.1 & 0.2 & nd & nd & nd & nd & nd & nd & nd & nd \\
\hline Benzo(ghi)perylene & 0.0 & 2.5 & 0.8 & 0.7 & nd & nd & nd & nd & nd & nd & nd & nd \\
\hline Anthanthrene & 0.0 & 0.7 & 0.1 & 0.2 & nd & nd & nd & nd & nd & nd & nd & nd \\
\hline Coronene & 0.0 & 1.9 & 0.4 & 0.6 & nd & nd & nd & nd & nd & nd & nd & nd \\
\hline Dibenzo(a,e)pyrene & 0.0 & 1.7 & 0.3 & 0.5 & nd & nd & nd & nd & nd & nd & nd & nd \\
\hline Subtotal & 4 & 54 & 24 & 15 & nd & nd & nd & nd & nd & nd & nd & nd \\
\hline \multicolumn{13}{|l|}{ VIII. Aromatic acids } \\
\hline 1,2-Benzenedicarboxylic acid & 2.9 & 53.8 & 13.2 & 13.1 & 3.1 & 31.7 & 12.8 & 9.6 & 3.1 & 73.4 & 16.5 & 17.9 \\
\hline 1,3-Benzenedicarboxylic acid & 0.5 & 5.1 & 1.2 & 1.1 & 0.2 & 6.2 & 1.8 & 1.9 & 0.0 & 2.7 & 0.9 & 0.9 \\
\hline 1,4-Benzenedicarboxylic acid & 0.6 & 16.8 & 3.6 & 4.2 & 0.7 & 6.0 & 2.7 & 1.8 & 0.5 & 18.8 & 3.7 & 4.9 \\
\hline Subtotal & 4 & 76 & 18 & 18 & 4 & 42 & 17 & 13 & 4 & 93 & 21 & 23 \\
\hline Total & 262 & 860 & 474 & 174 & 145 & 689 & 410 & 180 & 99 & 831 & 337 & 245 \\
\hline
\end{tabular}



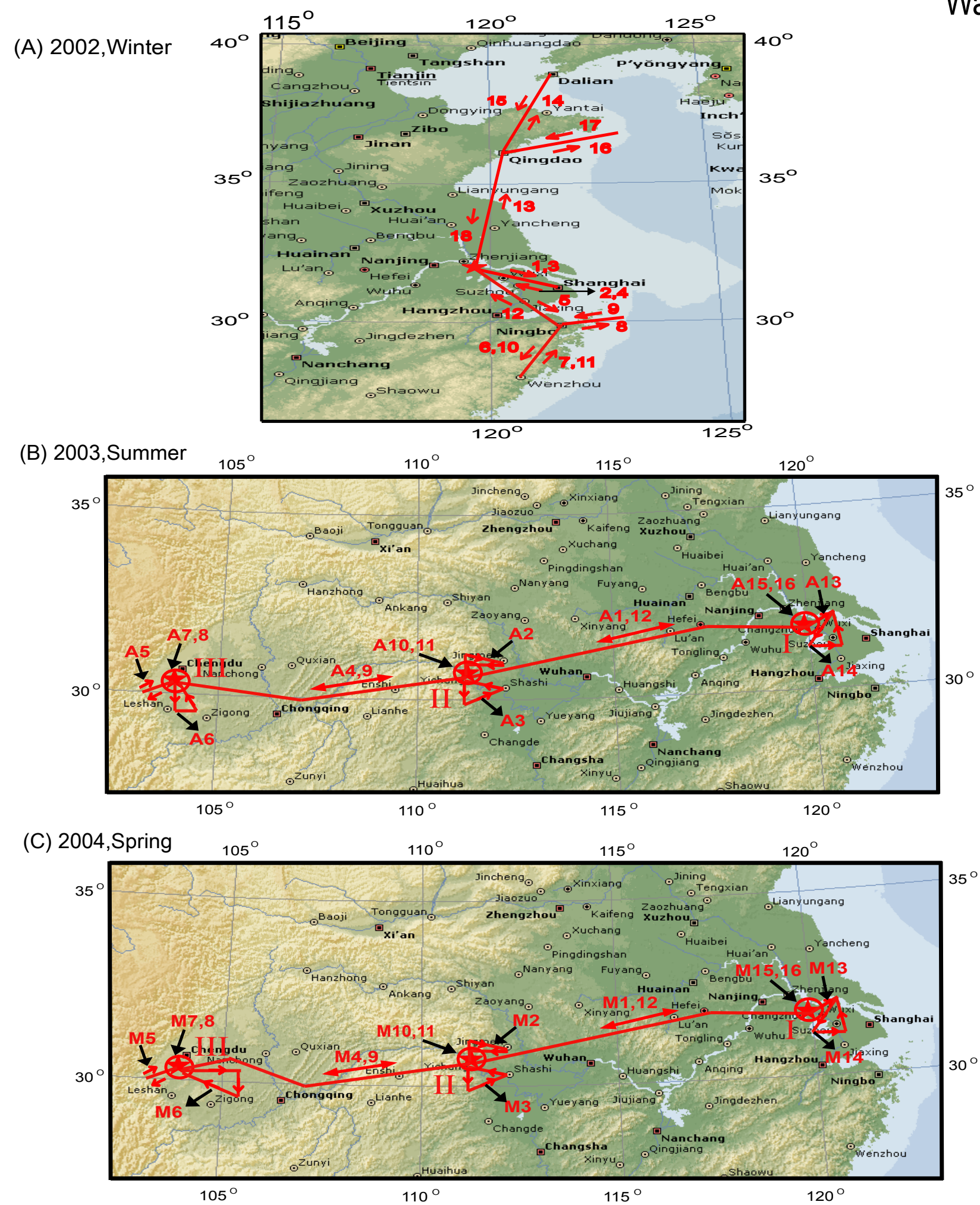

Figure S1. Flight tracks during the three campaingns (cities with population more than one million are shown as squares, and others are shown as circles). 18 Flights carried out for the winter campaign, while 16 flights carried out in each season during the summer and spring campaings. Aerosol sample was continuously collected throughtout each flight, resulting in 18, 16, and 16 PM2.5 samples for the winter, summer and spring campaigns, respectively. However, the summer PM 2.5 samples A9 and A12 and the spring PM2.5 sample M12 were used for other studies and not included in this study. Flights $A 7,8, A 10,11$, and $A 15,16$ in the figure $B$ and $M 7,8, M 10,11$, and $M 15,16$ in the figure $C$ were hovering flights over the local cities and shown in the figures as circles (named as O-shape flight). Flights A2, A3, A5, A6, A13 and A14 in the figure $B$ and flights M2, M3, M5, M6, M13 and M14 in the figure $C$ were also designed for the local cities (named as L-shape flight). Concentrations of organic aerosols from both kinds of local flights were averaged for their local abundances, while concentraions of organic compounds in the samples collected during the round flights between two cities were averaged and shown as a column in the middle of the cities.

Due to no $\mathrm{O}$ - or L-shape flight in the winter campain, concentraions of organic aerosols collected in round flights between two cities were averaged and charted as a column in the middle of the two cities to show their spatial distributions (Spatial distributions of organic aerosols shown in Figure 1). 